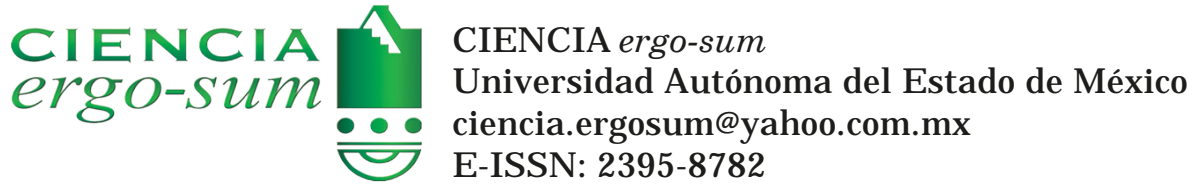

\title{
Los enviados de Belcebú: moscas y mosquitos (Diptera) exóticos con interés médico y veterinario en México
}

I báñez-Bernal, Sergio; Sandoval-Ruiz, César A.

Los enviados de Belcebú: moscas y mosquitos (Diptera) exóticos con interés médico y veterinario en México

CIENCIA ergo-sum, vol. 28, núm. 1, marzo-junio 2021 |e114

Universidad Autónoma del Estado de México, México

Esta obra está bajo una Licencia Creative Commons Atribución-NoComercial-SinDerivar 4.0 Internacional .

I báñez-Bernal, S. y Sandoval-Ruiz, C. A. (2021). Los enviados de Bel cebú: moscas y mosquitos (Diptera) exóticos con interés médico y veterinario en México. CIE NCIA ergo-sum, 28(1). https://doi.org/10.30878/ces.v28n1a8 


\title{
Los enviados de Belcebú: moscas y mosquitos (Diptera) exóticos con interés médico y veterinario en México
}

\section{Beelzebub's envoys: Exotic flies and mosquitoes (Diptera) of medical and veterinary interest in Mexico}

\author{
Sergio Ibáñez-Bernal \\ Red Ambiente y Sustentabilidad, Instituto de Ecología, A. C., México \\ sergio.ibanez@inecol.mx \\ (D) http://orcid.org/0000-0002-3182-6134 \\ César A. Sandoval-Ruiz \\ Facultad de Ciencias Biológicas, Benemérita Universidad Autónoma de Puebla, México \\ cesar.sandoval@correo.buap.mx \\ (1) https://orcid.org/0000-0002-8322-5525
}

Recepción: 12 de agosto de 2019

Aprobación: 28 de mayo de 2020

Escucha el ruido de sus alas... Nos rodean... Nos acechan:
dentro de un instante caerán sobre nosotros y sentiré
mil patas pegajosas sobre mi cuerpo... ¿A dónde huir?
Se hinchan, se hinchan, ya son grandes como abejas,
nos seguirán a todas partes en espesos remolinos.
¡Horror! Veo sus ojos, sus millones de ojos que nos miran"

(Sartre, 1947, 92)

\section{RESUMEN}

Se informan las razones por las que una especie puede introducirse en nuevas áreas geográficas y el impacto que tiene su presencia en los nuevos ambientes y su implicación para el bienestar humano. Los insectos exóticos del orden Diptera tienen implicaciones sociales y económicas considerables. Se presenta una lista preliminar de 22 especies de moscas y mosquitos exóticos con interés médico y veterinario que se encuentran en el territorio mexicano. Se comentan las posibles consecuencias en la salud, la economía y la conservación de los ambientes naturales. Se enfatiza la importancia de la taxonomía, de los estudios faunísticos y de la vigilancia entomológica en el territorio con especial énfasis en las fronteras para su detección, combate o restringir su distribución.

Palabras Clave: fauna exótica, especies invasoras, insectos vectores, insectos parásitos.

\begin{abstract}
We present the main reasons involved in the introduction of species into new geographical areas, and the impact of their presence in the environment, ecosystems and human welfare. Exotic insects of order Diptera are extremely important from the social and economic point of view. A preliminary commented list of 22 species of invasive flies and mosquitoes of medical and veterinary importance in Mexico, and their possible impacts to health, economy and ecosystem conservation is presented. We explain the importance of taxonomy, faunistic studies, and entomological surveillance in the country, with special interest on borders to detect, control or limit their distributions.
\end{abstract}

KEYWORDS: Exotic fauna, invasive species, vector species, parasitic insects.

\section{INTRODUCCIÓN}

La relación que existe entre moscas y mosquitos con el humano y sus animales domésticos precede cualquier evidencia histórica. Las moscas y mosquitos conforman el orden Diptera, un grupo de insectos que se caracteriza porque los adultos presentan el primer par de alas membranosas y funcionales para el vuelo, mientras que el segundo 
par de alas está modificado a manera de pequeños bastos con función sensorial durante el vuelo. El registro fósil de dípteros más antiguo procede de entre el Pérmico tardío y el Triásico temprano, hace aproximadamente 250 millones de años (Grimaldi y Engel, 2005; Wiegmann et al., 2011), por lo que anteceden a la especie humana por al menos 248 millones de años.

En general, los dípteros adultos se alimentan de materia orgánica en descomposición, pero existen muchas especies con hábitos depredadores, parasitoides, parásitos y muchas otras que se alimentan de plantas (Hövemeyer, 2000). Los adultos en general son excelentes voladores y suelen encontrarse cerca de los lugares donde están los recursos para alimentarse y donde las etapas juveniles pueden desarrollarse. Como los dípteros tienen un desarrollo holometábolo (metamorfosis completa con etapa juvenil larvaria, etapa intermedia de pupa y etapa adulta), las larvas suelen hacer uso de recursos alimentarios y ambientes diferentes a los de los adultos, con juveniles acuáticos, semiacuáticos o en hábitats secos. Debido a esta gran variedad de estilos de vida, existen especies en prácticamente todos los ambientes del mundo, con la excepción de los océanos, los polos y los picos montañosos con nieve perpetua (Brown et al., 2009). Los dípteros son además esenciales para la adecuada funcionalidad de los ecosistemas en donde ocurren.

A la fecha se reconoce que el orden Diptera es uno de los grupos de insectos más diversos y exitosos con más de 159000 especies descritas pertenecientes a 158 familias en el mundo (Pape et al., 2011), solamente superado por los escarabajos (orden Coleoptera) y las polillas, palomillas y mariposas (orden Lepidoptera). Sin embargo, el estudio del orden Diptera no ha sido tan intenso como con los otros grupos de insectos mencionados, por lo que se estima que debe existir al menos el doble de especies (Borkent et al., 2018). A pesar de que más de $80 \%$ de especies se consideran benéficas por su papel en los ecosistemas como componentes elementales de las redes de alimentación como descomponedores, alimento de arácnidos, insectos, peces, anfibios, reptiles, aves, mamíferos, además de tener relaciones importantes como polinizadores, parásitos y parasitoides que regulan poblaciones de otros organismos (Pape, 2009), este grupo de insectos es conocido por una cantidad proporcionalmente pequeña de especies que inciden de manera negativa en la salud del humano y de los animales domésticos.

Dentro de las familias de dípteros mejor estudiadas se encuentran aquellas parásitas de vertebrados que incluyen las que se alimentan de sangre debido a la gran importancia que tienen como plagas sanitarias y sobre todo por su papel en la transmisión de organismos patógenos que producen enfermedades en el humano y en animales domésticos, por lo que adquieren gran importancia social con fuertes consecuencias económicas. Por mencionar un ejemplo de su importancia médica, dos especies de mosquitos transmiten el virus del dengue en las zonas tropicales y subtropicales del mundo, lo que causa alrededor de 390 millones de infecciones al año y de las cuales alrededor de 96 millones tienen manifestaciones clínicas (Bhatt et al., 2013), además de que se calcula que 3.9 mil millones de personas viven en zonas de riesgo de transmisión (Brady et al., 2012; WHO, 2019).

Las especies de dípteros con implicaciones en la salud humana y de sus animales domésticos se restringen principalmente a unas cuantas familias: Culicidae (mosquitos, moyotes o zancudos), Psychodidae-Phlebotominae (papalotillas), Ceratopogonidae (jejenes), Simuliidae (chaquistes de río), Tabanidae (tábanos o moscas de los caballos y venados), Faniidae (moscas menores), Muscidae (mosca doméstica y especies relacionadas), Calliphoridae (moscas metálicas), Oestridae (moscas del estro), Glossinidae (moscas tsé tsé) e Hippoboscidae (moscas planas). Cabe destacar que muchas de las especies con interés médico y veterinario que se han introducido en nuevos territorios en vastas regiones del mundo son especies exóticas relacionadas con el humano y sus animales domésticos.

Las especies consideradas sinantrópicas son aquellas que se asocian espacialmente y en el tiempo con el humano y el entorno donde él se encuentra, como también donde están sus animales domésticos (antropobiocenosis de acuerdo con Povolnỳ, 1971). Se pueden reconocer varios tipos de especies sinantrópicas, pero en esencia son importantes desde el punto de vista médico y veterinario las especies antropófilas (modificado de Di Castri et al., 1990), aquellas que aprovechan al humano de forma directa o que se adaptan a las condiciones creadas por sus asentamientos o sus actividades y obtienen los recursos para desarrollarse y sustentar sus poblaciones a través del tiempo. Además, dentro de las especies antropófilas se pueden reconocer como especies kairófilas aquellas que 
se relacionan con él o con su entorno primariamente por ser un recurso natural que siempre ha aprovechado el insecto y como neófilas aquellas que secundariamente se relacionan con el humano por ser un recurso potencial disponible o por encontrar condiciones adecuadas a causa de la actividad humana (cambios antropogénicos), en especial por la alteración de los ambientes naturales para cultivos o para ganado (agrobiocenosis de acuerdo con Povolnỳ, 1971). Algunas de estas especies también pueden incorporarse a los ecosistemas naturales (eubiocenosis según con Povolnỳ, 1971) con implicaciones dañinas para las comunidades biológicas nativas; los efectos de las especies invasoras en los ecosistemas naturales son muy difíciles de valorar.

Las especies que se encuentran en las diversas regiones biogeográficas, áreas fisiográficas y ecosistemas son diferentes y conforman ensamblajes únicos limitados por barreras geográficas y que por sus atributos particulares intrínsecos no pueden sobrepasar naturalmente. La distribución natural de las especies cambia, entre otras razones, si hay vías artificiales que les permitan rebasar esos límites para encontrar en otros sitios condiciones adecuadas para establecerse y sobrevivir como sería el clima, recursos para refugio, alimentación, así como la inexistencia de parásitos, parasitoides y depredadores naturales que regulen la abundancia de sus poblaciones, además de otros factores.

Las especies de dípteros exóticos con interés médico y veterinario son en su mayoría sinantrópicas y antropófilas, que alcanzan áreas geográficas donde original y naturalmente no existían y que en los nuevos territorios encuentran condiciones propicias para su subsistencia a las cuales se adecúan muy rápido colonizando y procreando en lo sucesivo generaciones viables. Si estas especies sobreviven, colonizan y se reproducen en los ecosistemas naturales de las áreas geográficas donde se introdujeron, se consideran especies invasoras (CONABIO, 2019).

\section{Principales motivos de la introducción de esPecies}

Cuando el humano comenzó a dispersarse para colonizar nuevas áreas geográficas, varias especies de plantas y animales fueron introducidas exprofeso para continuar siendo utilizadas como recurso. Además, con ellas pudieron introducirse con facilidad otros organismos no deseados sin ser percibidos. Con las personas, por ejemplo, se introdujeron diversos parásitos kairófilos que incluyen insectos como piojos o con la fauna sinantrópica o doméstica, como ratas y perros pulgas, garrapatas, etc.; con el ganado, diversas moscas y otros parásitos kairófilos y neófilos; con los reservorios de agua potable, algunas especies de mosquitos, así como también con las provisiones y mercancías, varias especies de cucarachas, moscas, entre otras.

En lo que va de este siglo, la globalización o integración mundial de los procesos políticos, económicos, tecnológicos, sociales y culturales ha ido minimizando las barreras que constituían las fronteras entre los países y ha ampliado la interacción de los mercados. La globalización también ha favorecido la eficacia de las comunicaciones y la rápida movilidad de las personas y productos comerciales entre diversos países al tiempo que la migración humana ha aumentado (Expósito Martín, 2012); todas lo anterior ha favorecido la introducción de fauna exótica. La modificación o la pérdida de los hábitats naturales por acción humana y el cambio climático son factores que afectan la integridad ecosistémica que favorecen el establecimiento de especies exóticas invasoras (Ibáñez-Bernal, 2012).

\section{IMPLICACIONES DE LA INTRODUCCIÓN DE ESPECIES A NUEVAS ÁREAS}

Las especies exóticas que se establecen en nuevos territorios pueden hacerlo en diversos ambientes con efectos y consecuencias diferentes:

a) En ambientes alterados que constituyen los asentamientos humanos. En este caso las especies exóticas se incorporan a las viviendas, en ciudades, pueblos y áreas rurales (antropobiocenosis), y se les considera eusinantrópicas (Povolnỳ, 1971). En este tipo de ambientes se ha eliminado en gran proporción las comunidades bióticas nativas. Las construcciones humanas por sí mismas representan un espacio con temperatura 
y humedad relativamente constantes y adecuadas que permiten el desarrollo de especies que de otra forma no podrían subsistir en el entorno. Desde luego, quedan nichos vacíos creados por la eliminación de flora y fauna nativas y son lugares donde existen recursos de alimento, refugio y donde no existen o son escasos los depredadores, parasitoides, parásitos, etc., que limiten o controlen sus poblaciones.

b) En ambientes alterados para la obtención de recursos para consumo o uso humano. Las especies exóticas se incorporan a la comunidad biótica en sitios donde se cultivan plantas o se cría ganado (agrobiocenosis) y se conocen como especies hemisinantrópicas (Povolnỳ, 1971). Como en el caso anterior, pero en menor medida, muchas especies de la comunidad biótica nativa se sustituyen por cultivos de plantas útiles para el humano, casi siempre en forma de monocultivos. En el caso de la ganadería, también se elimina gran parte de la flora y su fauna acompañante nativa para permitir el desarrollo de pastizales que provean de alimento al ganado (vacuno, equino, caprino, ovino, etc.) que también es introducido a esas zonas. En estos lugares se establecen muchas especies exóticas de parásitos que aprovechan de alguna manera a los animales, sus productos o sus desechos (heces, orina, cadáveres, etc.), que también son recursos útiles con poca competencia por parte de especies nativas.

c) En ambientes naturales. Las especies exóticas se incorporan a las comunidades de organismos nativos (eubiocenosis), las cuales se consideran especies asinantrópicas (Povolnỳ, 1971) y por tanto invasoras. Suelen ser especies con atributos intrínsecos que les permiten adaptarse con facilidad a las condiciones de un ecosistema, aprovechan recursos disponibles en el medio con gran facilidad, sus poblaciones pueden crecer rápidamente en forma desmedida por carecer de enemigos naturales y compiten por recursos con especies nativas al grado de desplazarlas o eliminarlas. Esto último conduce a la pérdida de biodiversidad no sólo por la competencia por los recursos, sino también con efecto dominó en la red trófica, lo que desestabiliza el funcionamiento normal del ecosistema con efectos de pérdida a nivel de gremio trófico e incluso también en un nivel de producción primaria, de consumidores primarios y secundarios.

Cabe indicar que ciertas especies exóticas podrían desarrollarse en todos los tipos de ambientes descritos.

\section{Criterio para Determinar si una ESPECIE ES eXótica}

En principio es necesario establecer la escala geográfica a la cual se referirá y el enfoque o criterio aplicado, el cual puede ser desde el punto de vista bioecológico o bien en un contexto geopolítico (antropocéntrico) que es consecuencia de la primera. Las introducciones bioecológicas son analizadas o referidas de acuerdo con la región biogeográfica (Neártica, Neotropical, Paleártica, Oriental, Arotropical, Australasia), por continentes, región fisiográfica, de ecosistemas, o de microhábitats, mientras que desde el punto de vista antropocéntrico los escenarios pueden ser de comunidades políticas, regiones económicas, país, estado, municipio o de asentamientos urbanos.

Para detectar una especie exótica debe tenerse un conocimiento amplio de la historia natural y la taxonomía de los grupos bajo estudio. Poder determinar la especie, distinguir una de otra sin importar la región geográfica del mundo, es fundamental y eso requiere de un conocimiento taxonómico amplio. Es necesario también contar con información faunística del área de detección con inventarios de especies lo más completos y fiables posibles. Resulta también indispensable contar con datos de la distribución geográfica de las especies.

Otro aspecto que debe adicionarse para discriminar si las especies exóticas de dípteros con interés médico y veterinario son invasoras, es el grado de sinantropía. Se ha desarrollado un indicador o índice de sinantropía (Nourteva, 1963) para calcular el grado de relación que guarda una especie en comparación con otros miembros del ensamblaje de especies del mismo taxón respecto a los diferentes grados de urbanización o modificación del ambiente natural. Este indicador basado en un levantamiento faunístico sistemático y periódico permite generar información sobre la relación que existe entre cada una de las especies de un ensamblaje con el tipo de ecosistema (León Montoya et al., 2009). 
En conclusión, para detectar especies exóticas son necesarios conocimientos en los ámbitos de la taxonomía y faunística, independientemente de contar con estrategias transversales de detección en fronteras puertos marítimos o aéreos que involucra no sólo los organismos operativos con estas funciones, sino una adecuada articulación con los institutos científicos y contar con el apoyo de especialistas en el ramo de la biología conocedores de los grupos biológicos involucrados. Para considerar su potencial como especies invasoras, deben analizarse, además de lo comentado, el grado en el que una especie ha colonizado los hábitats naturales, realizar estudios ecológicos y detectar los posibles efectos de su presencia en la estructura y funcionalidad de la comunidad donde se encuentre.

\section{Principales especies exóticas de dípteros con interés médico y Veterinario en MéXico}

La lista de IUCN (2019) sólo incluye dos especies de Diptera de la familia Culicidae exóticas en México. La relación de especies en el Acuerdo por el que se determina la lista de especies exóticas invasoras para México (Diario Oficial de la Federación, 2016) incluye cuatro especies de Cecidomyiidae (con interés agrícola) y una especie de mosquito Culicidae.

En este trabajo se da conocer una lista de dípteros exóticos de México enfocada en las familias que son hematófagas, que son parásitos de vertebrados o que tienen capacidad de transmitir mecánicamente patógenos y que, por lo tanto, tienen importancia en salud pública y animal.

La lista que a continuación se presenta está basada en información obtenida por Sergio Ibáñez-Bernal durante la elaboración del Catálogo de Autoridad Taxonómica del orden Diptera (Insecta), bajo el apoyo y financiamiento de CONABIO, el cual está dividido en tres partes: a) Parte 1. Suborden Nematocera. (CONABIO, CS004).b) Parte 2. Suborden Brachycera Inferiores. (CONABIO ES0011).c) Parte 3). Actualización del Catálogo de Autoridad Taxonómica del orden Diptera (Insecta) de México (JE006), que está reforzado mediante valoraciones de las afinidades filogenéticas y de la distribución geográfica de las especies de cada género.

Es importante recalcar la importancia de indicar cuáles son las especies exóticas presentes en México como primer paso para posteriormente determinar la capacidad invasora de cada una de ellas.

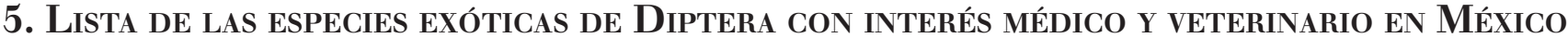

\section{1. Familia Culicidae (mosquitos, moyotes, zancudos)}

\section{1. 1. Aedes (Stegomyia) aegypti (Linnaeus, 1762)}

Esta especie y Aedes albopictus (figura 1) son especies que forman parte del subgénero Stegomyia, el cual agrupa 128 especies distribuidas en su mayoría en la región Oriental, Africana, Islas del Pacífico y Australiana. Aedes aegypti puede considerarse a la fecha cosmopolita; aparentemente, fue distribuida por el mundo desde tiempos de las expediciones comerciales y de conquista tanto en los reservorios de agua como en contenedores vacíos, ya que los huevos pueden entrar en diapausa y soportar varios meses sin el líquido. En México, se comporta como eusinantrópica y puede encontrarse en ciudades y poblados. Se le relaciona con viviendas donde hay contenedores artificiales con agua de lluvia y almacenada, e incluso en zonas áridas y semiáridas en recipientes donde la gente acumula agua para su uso doméstico. A pesar del ambiente donde la encontramos, puede alimentarse de animales domésticos además del humano. Es vector de virus de la fiebre amarilla (en ciclo urbano), del dengue, del virus chikungunya, del virus zika, mayaro, encefalitis equina del este, fiebre del virus del Oeste del Nilo, encefalitis equina venezolana, entre otros (Larsen y Ashley, 1971). 


\section{1. 2. Aedes (Stegomyia) albopictus (Skuse, 1894)}

Esta especie es la única de díptero con interés médico considerada en el Acuerdo por el que se determina la Lista de Especies Exóticas Invasoras en México (Diario Oficial de la Federación, 2016). Es una especie de origen asiático que llegó a la ciudad de Houston, Estados Unidos, en 1985; aparentemente sus huevos fueron transportados y también pueden suspender su desarrollo en diapausa en neumáticos usados desde Japón (Hawley et al., 1987). Su expansión fue lenta al principio en los Estados Unidos, pero ha colonizado varios estados del país vecino. Hacia 1994 se detectó en la frontera de Tamaulipas (Ibáñez-Bernal \& Martínez-Campos, 1994) y a la fecha su distribución abarca al menos nueve estados. A diferencia de la especie anterior, en México Ae. albopictus es eusinantrópica y hemisinantrópica, por lo que suele encontrarse en microhábitats junto con Aedes aegypti, pero también en otros sitios en las afueras de las poblaciones donde Ae. aegypti no lo está. Se considera vector del virus dengue, zika, chikungunya, fiebre amarilla, virus del Oeste del Nilo, virus de la encefalitis japonesa, virus Rift Valley, encefalitis equina y de filarias del género Dirofilaria, entre otros patógenos (Hawley, 1988).

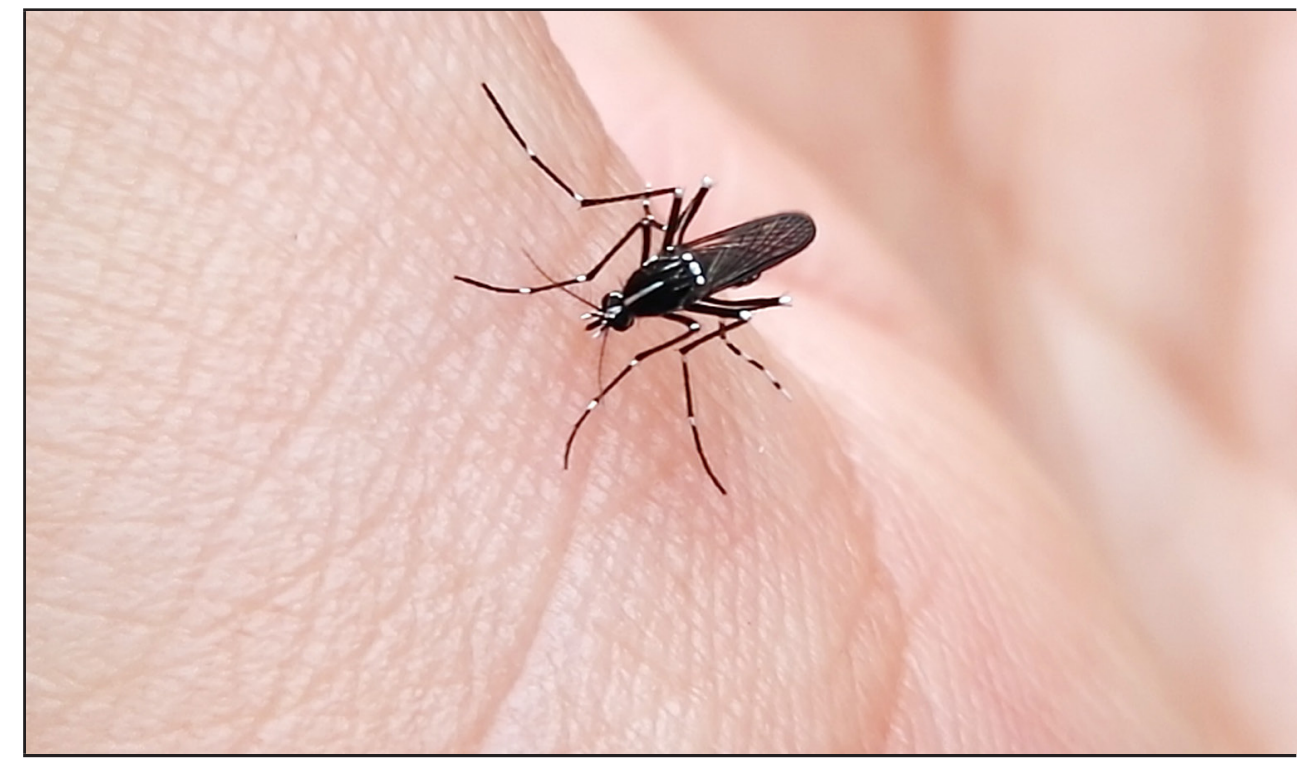

FIGURA 1

Aedes albopictus (Diptera: Culicidae)

Fuente: foto de César A. Sandoval-Ruiz.

\section{2. Familia Psychodidae (mosquitos mariposa, papalotillas)}

Se reconocen seis subfamilias, una de las cuales (subfamilia Phlebotominae) tiene importancia médica por incluir especies hematófagas de vertebrados (Wagner e Ibáñez-Bernal, 2009). El resto de las subfamilias carece de interés directo para el humano, excepto por ciertas especies de la subfamilia Psychodinae, que son eusinantrópicas y se han dispersado por el mundo. Las especies que se enlistan abajo son exóticas en México y suelen encontrarse en los baños, cocinas, tuberías de drenaje y áreas muy húmedas de las construcciones, donde incluso los individuos pueden ser tan numerosos que se consideran especies plaga de tipo doméstico o de cierto tipo de industrias que cuenten con material orgánico en descomposición donde crecen bacterias y hongos. Son especies con gran potencial para estimar el grado de perturbación de los ecosistemas. Existen algunos informes de ellas invadiendo los conductos gástricos y urogenitales del humano, pero este tipo de miasis es más bien una infestación fortuita (James, 1947; Zumpt, 1965). 


\section{2. 1. Psychoda (Tinearia) alternata (Say, 1824)}

Las especies del subgénero Tinearia son aparentemente holárticas en origen. Esta especie es cosmopolita en la actualidad y también se ha dispersado siguiendo al humano por el mundo. Se encuentra con certeza en todo México, pero existen muy pocos trabajos que permitan confirmarlo (Ibáñez-Bernal, 2000; Ibáñez-Bernal, 2008). Los estudios faunísticos que hemos realizado concuerdan en indicar que en México se comporta como eusinantrópica, ya que conforme uno se aleja de la construcción humana la especie va escaseando hasta desaparecer.

\section{2. 2. Psychoda cinerea (Banks, 1894)}

Se considera cosmopolita y ha sido registrada en las regiones biogeográficas Neártica, Neotropical, Paleártica y Australiana. En México se encuentra bajo las mismas condiciones eusinantrópicas y hemisinantrópicas que las descritas para Psychoda alternata (Ibáñez-Bernal, 2008).

\section{2. 3. Clogmia albipunctata (Williston, 1893)}

Esta especie, considerada por algunos autores como Telmatoscopus albipunctatus, en cuyo caso Clogmia es considerado como subgénero de Telmatoscopus, es una especie actualmente pantropical con registros en las áreas biogeográficas Neotropical, Neártica, Paleártica, Africana, Oriental y Australiana. También es una especie con potencial para indicar el grado de perturbación ecosistémica y en México se encuentra bajo las mismas condiciones que las especies de Psychoda mencionadas (Ibáñez-Bernal, 2008).

\section{3. Familia Faniidae (moscas menores)}

\section{3. 1. Fannia canicularis (Linnaeus, 1761)}

Esta especie se considera hoy día cosmopolita, siendo sinantrópica y hemisinantrópica (Chillcott, 1961), ya que se ha registrado en el área Paleártica, Neártica, Neotropical, Australiana, norte de África, Hawai, etc. Es transmisora mecánica de Escherichia coli y Staphylococcus aureus, protozoarios y nematodos (Rozkošný et al. 1997), además de que se ha registrado como miasígena (James, 1947).

\subsection{Familia Muscidae (moscas comunes o domésticas)}

\section{4. 1. Haematobia irritans (Linnaeus, 1758)}

Esta especie aparentemente paleártica es en la actualidad cosmopolita (Hogsette y Farkas, 2000). En México se encuentra asociada sobre todo al ganado vacuno y equino en ambientes hemisinantrópicos. Suele constituir una plaga sanitaria que afecta la adecuada alimentación de los animales, además que ha sido relacionada con la transmisión de ciertos patógenos como Staphylococcus aureus (Gillespie et al., 1999), entre otros.

\section{4. 2. Musca domestica (Linnaeus, 1758)}

Esta especie es hoy cosmopolita y fuertemente sinantrópica y hemisinantrópica, y se encuentra distribuida en todo México. Se ha registrado como productora de miasis (James, 1947) y puede transmitir de forma mecánica diversos patógenos debido a sus hábitos alimentarios eclécticos. 


\section{4. 3. Muscina stabulans (Fallén, 1817)}

A la fecha, esta especie se considera que tiene una gran distribución en el mundo con registros en las áreas Paleártica, norte de África, Oriental, Neártica y Neotropical. Es una especie exótica en México, Venezuela, Brasil, Uruguay, Argentina y Chile (Pape y Evenhuis, 2019).

\section{4. 4. Stomoxys calcitrans (Linnaeus, 1758)}

Esta especie aparentemente es de origen euroasiático (Bishop, 1913) y puede encontrarse en las áreas Neártica, Neotropical, Paleártica, Oriental y Australiana. En América se encuentra desde Alaska y Columbia Británica hasta California y Florida en los Estados Unidos, México, Centroamérica y Sudamérica. En México posee gran distribución y se encuentra como especie hemisinantrópica; con frecuencia se le relaciona con el ganado estabulado. Es una especie donde machos y hembras son hematófagos, y pudiera estar involucrada en la transmisión de diversos patógenos al ganado (Greenberg, 1973).

\section{5. Familia Calliphoridae (moscas metálicas)}

\section{5. 1. Calliphora grahami (Aldrich, 1930)}

Esta especie se distribuía originalmente en las áreas Paleártica y Oriental, pero fue introducida en los Estados Unidos y de allí se dispersó en México (Whitworth, 2006). No se ha relacionado todavía con algún agente patógeno.

\section{5. 2. Calliphora vicina (Robineau-Desvoidy, 1830)}

Especie originaria de la región Holártica que se ha dispersado a las regiones Oriental, Australiana e incluso en el área Neotropical. Es una especie detritívora y en ocasiones se ha encontrado causando miasis (Zumpt, 1965).

\section{5. 3. Chrysomya rufifacies (Macquart, 1843)}

Es una especie originaria de Australasia. Se informa de ella por primera vez en América con ejemplares capturados en Costa Rica en 1978 y que para 1988 ya se encontraba distribuida hasta el norte de México (Greenberg, 1988). A la fecha esta especie ya se considera cosmopolita (Dear, 1985). Es una especie miasígena secundaria que afecta ovejas y otros animales domésticos (James, 1947).

\section{5. 4. Chrysomya megacephala (Fabricius, 1784)}

En sus orígenes esta especie tenía una distribución en las áreas Oriental y Australiana (Greenberg, 1973). Es invasora en México y se asocia principalmente con las zonas urbanas de casi todo el país (Greenberg, 1988). Se considera hoy día cosmopolita; su distribución se ha expandido desde Brasil hasta Estados Unidos de Norte América (Silva et al., 2012). Es una especie saprófaga, pero se ha informado como miasígena secundaria de lesiones en la piel, aberturas corporales y senos respiratorios (James, 1947)

\section{5. 5. Lucilia cuprina (Wiedemann, 1830)}

Es una especie común en heces de animales domésticos, en materia vegetal en descomposición, tiraderos de basura y en puestos callejeros de comida. A la fecha se considera cosmopolita y en América se encuentra desde 
Estados Unidos hasta Argentina (Whitworth, 2014). En México parece comportarse como eusinantrópica y hemisinantrópica.

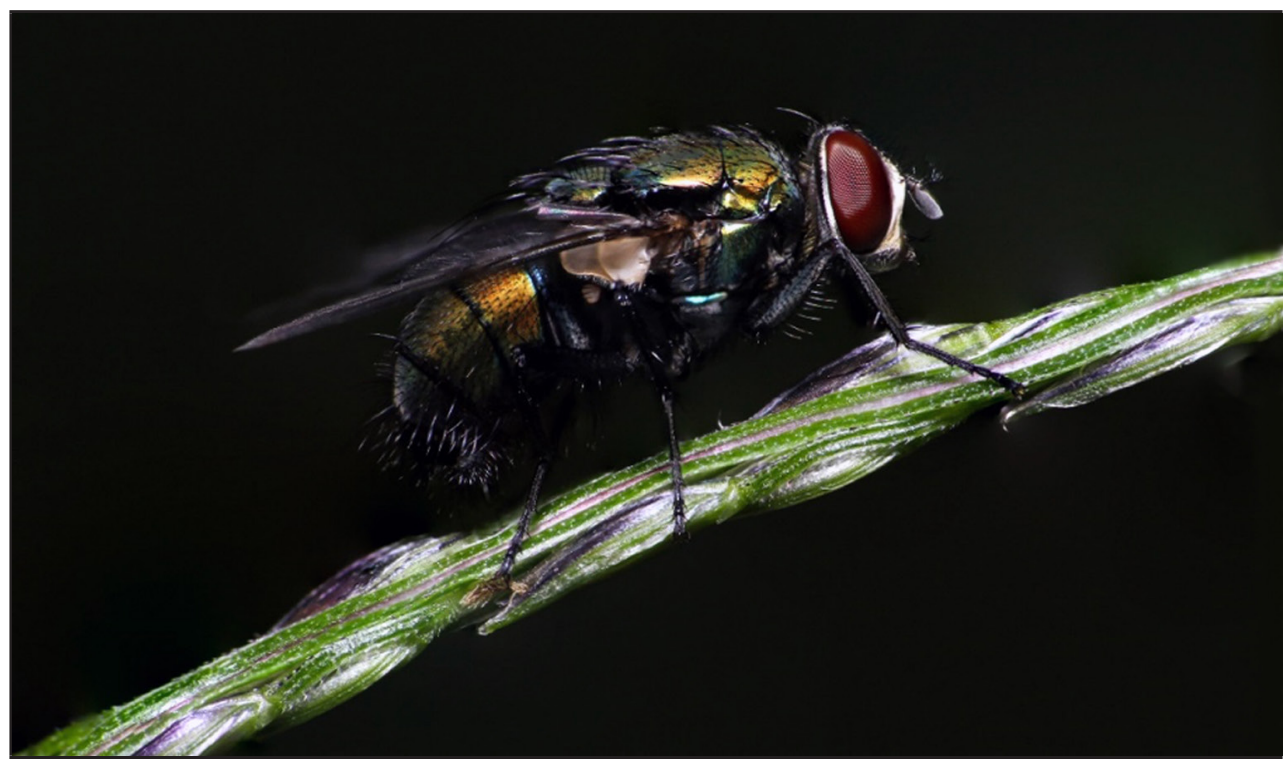

FIGURA 2

Lucilia cuprina (Calliphoridae)

Fuente: foto de Santiago Jaume-Schinkel.

\section{5. 6. Lucilia sericata (Meigen, 1826)}

Aparentemente holártica en origen, ahora es cosmopolita (James, 1947; Greenberg, 1973) y parece ser eusinantrópica y hemisinantrópica en México. Se alimenta de materia orgánica en descomposición y heces. Puede funcionar como vector mecánico de diversos organismos patógenos y se ha informado como miasígena en México (Martínez-Rojano et al., 2018).

\section{5. 7. Phormia regina (Meigen, 1826)}

Especie holártica frecuente en regiones frías, que se distribuye en Norteamérica y también en México (James, 1947). Está involucrada en miasis de heridas de animales domésticos, aunque es principalmente saprófaga (Greenberg, 1973).

\section{6. Familia Oestridae (moscas del estro)}

\section{6. 1. Gasterophilus haemorrhoidalis (Linnaeus, 1758)}

Aparentemente de origen holártico, a la fecha es una especie cosmopolita. Su hospedero común es el caballo; las larvas infestan su región oral, pero después migran al estómago (James, 1947; Yan et al., 2019).

\section{6. 2. Gasterophilus intestinalis (De Geer, 1776)}

$\mathrm{Al}$ igual que la anterior, esta especie es de origen holártico y a la fecha se considera cosmopolita. Su hospedero común es el caballo las larvas infestan su región oral, y también cuenta con registros de infestación en humanos (James, 1947; Yan et al., 2019). 


\section{6. 3. Gasterophilus nasalis (Linnaeus, 1758)}

Con un posible origen holártico, a la fecha es una especie cosmopolita, aunque poco frecuente en el área neotropical en comparación con las otras dos especies del género (Yan et al., 2019).

\section{6. 4. Oestrus ovis (Linnaeus, 1758)}

Esta especie tiene actualmente una distribución mundial. Es parásita en estado larval de diversos mamíferos que afecta a los animales domésticos y en ocasiones también al humano (James, 1947).

\section{7. Familia Hippoboscidae (moscas planas)}

\section{7. 1. Melophagus ovinus (Linnaeus, 1758)}

Especie paleártica de Europa, noroeste de África, Mongolia, norte de la India y en la actualidad ya introducida en América, la región Australiana y Oriental (Pape y Evenhuis, 2019). Es parásita en especial de ovinos (Larroza, 2013).

\section{ANÁlisis PROSPECTIVO}

Es tal el impacto mundial de las especies invasoras que la Organización de las Naciones Unidas encabeza el Programa Global de Especies Invasoras (GISP, por sus siglas en inglés). Relacionado con dicho programa, en México existe una agenda de trabajo en el marco de biodiversidad de la Comisión de Cooperación Ambiental para América del Norte en el ámbito del Tratado de Libre Comercio para América del Norte (TLCAN) (Secretariado de la Comisión para la Cooperación Ambiental, 1993). La estrategia nacional sobre especies invasoras en México propone la consolidación de sistemas de prevención, control y eliminación mediante la coordinación participativa de instituciones y sectores sociales clave que incidan en aspectos legislativos y normativos, así como en la capacidad científica para su detección, seguimiento y coordinación de las acciones para atender problemas relacionados con estas especies (Comité Asesor Nacional sobre Especies Invasoras, 2010).

Las especies exóticas pueden ser separadas en dos grupos generales que merecen un tratamiento diferente: $a$ ) las especies con alto potencial de introducirse que, aunque no se han registrado, pueden haberse detectado en otras regiones, países cercanos o con los que se tienen relaciones comerciales o en fronteras terrestres, aéreas o marítimas y aduanas o almacenes de productos de importación y b) las especies kairófilas y neófilas que ya están presentes en el área de interés.

Respecto a las especies exóticas invasoras que potencialmente pueden introducirse y establecerse en ecosistemas, las acciones deben involucrar la detección temprana para reducir ese riesgo, así como gestionar programas de vigilancia y monitoreo de las poblaciones y procurar programas de control que eviten su establecimiento y dispersión (Comité Asesor Nacional sobre Especies Invasoras, 2010).

Es necesario contar con información actualizada y confiable sobre las especies exóticas ya presentes, sobre todo si son kairófilas y tienen mucho tiempo ocupando áreas geográficas que no les corresponden. Estas especies casi siempre pasan a ser cotidianas para el humano con el paso del tiempo y son tratadas por la gente como si fueran especies comunes para el lugar. No obstante, pueden ser especies problemáticas, algunas tan abundantes como para considerarse plagas, puesto que pueden implicar riesgos sanitarios o actuar en el deterioro de los productos y reducir su valor comercial. Estas especies por lo regular dejan de ser monitoreadas y solamente son tratadas por los particulares mediante algún método de control, por lo general químico. El uso de insecticidas para uso doméstico e incluso varios productos para uso agropecuario no están del todo regulados, por lo que su empleo, lejos de controlar el problema, lo complican al promover la resistencia a los pesticidas por parte de las especies 
exóticas al tiempo que deterioran las poblaciones de especies nativas que pudieran ser clave para el control natural de las primeras.

Estas especies deben ser monitoreadas para conocer su distribución geográfica y detectar cualquier expansión en áreas no ocupadas, incluso si hay cambios en sus hábitos como para incorporarse de una antropobiocenosis a una agrobiocenosis o hasta una eubiocenosis y para conocer la forma en la que interaccionan con otros miembros de las comunidades bióticas al considerar el grado de modificación de los ecosistemas, así como el deterioro de la funcionalidad de los ecosistemas por competencia por recursos o depredación y parasitismo (Wilcove et al., 1998). Es igual de imperante estudiar el papel en la transmisión de patógenos y explorar el posible efecto que sobre ellas tenga el cambio climático para hacer predicciones sobre el cambio de su distribución. Tampoco es común que se analice la importancia sanitaria y médico veterinaria de muchas de estas especies invasivas (Suaya et al., 2009), ni el impacto económico directo por daños en la salud, gastos médicos y en medicamentos, la inversión para aplicar métodos de control poblacional o estimaciones realistas de las pérdidas en productos o deterioro de su calidad (Pimentel et al., 2005).

Es de vital importancia contar con una lista de especies exóticas sobre las cuales realizar monitoreos sistemáticos para conocer su abundancia y distribución, además de detectar los efectos de su presencia en los diversos ecosistemas, los efectos directos en salud del humano y los animales domésticos y silvestres y las consecuencias económicas de su presencia.

La detección de nuevas especies exóticas y el seguimiento de las ya presentes en el territorio debe descansar en un amplio conocimiento taxonómico de los grupos en cuestión sin importar el área geográfica de la que se trate, ya que errores en la identificación o malas interpretaciones de las características que permiten reconocerlas puede resultar en registros falsos o en la ausencia de detección de dichas especies. Por desgracia, desde hace muchos años se ha dejado de lado en los programas educativos varios aspectos de biología descriptiva para dar paso a materias de biología comparativa. Sistemáticamente, se desprecian los estudios faunísticos y en cierta manera se ha dado preferencia al análisis de datos que, si bien son muchos, están incompletos si consideramos la escasez de estudios entomofaunísticos en diversas áreas geográficas y los cambios drásticos que los ecosistemas están experimentando por los efectos antropogénicos en lo que hoy se ha dado en llamar el Antropoceno. Una de las áreas más deterioradas de los planes de estudio en biología es la taxonomía. Debe entenderse que la taxonomía no implica como meta última nombrar especies, sino asociar las características morfológicas, biológicas, de distribución geográfica, ecológicas, genéticas, de comportamiento, etc., a esa entidad o especie nombrada de manera universal (Mayr, 1969).

Debe tenerse en cuenta la falta de conocimiento respecto a las especies de insectos presentes en México. Los dípteros están representados por al menos 101 de las 150 familias reconocidas en el mundo, pero hasta principios de 2015 se habían registrado sólo 6157 especies a pesar de que se estima que deben existir al menos 20000 especies si se considera que el país posee 10\% de la diversidad mundial (Ibáñez-Bernal, 2017).

En conjunto con la taxonomía, la elaboración de inventarios faunísticos adecuadamente estructurados para registrar las especies presentes en los diferentes ecosistemas o regiones fisiográficas bajo las diferentes condiciones climáticas del año es labor indispensable que permite el registro de las especies nativas y la detección de especies invasoras, información necesaria también para documentar los posibles cambios en la estructura de los ecosistemas. Estos aspectos del campo de la biología deben ser fomentados en las instituciones educativas y en los centros de investigación del país.

El abordaje de aspectos taxonómicos y faunísticos no se desarrolla adecuadamente sin la existencia de colecciones biológicas que permitan la consulta del acervo biológico de regiones, países y hasta zonas biogeográficas completas por parte de las nuevas generaciones.

Es muy difícil establecer programas de vigilancia robustos que permitan detectar la introducción de especies en un territorio sin la existencia de especialistas taxónomos, sin la procuración de estudios faunísticos y sin apoyo de las colecciones biológicas y de la información bibliográfica relacionada con ella. De igual forma, es necesario fortalecer los programas de detección de especies en las puertos, aeropuertos y fronteras, ya que deben conside- 
rarse como las vías primarias de entrada de especies exóticas, o bien de individuos de una especie previamente introducida, pero pertenecientes a otras poblaciones que con toda seguridad han estado sujetas a un historial de control o a otras presiones selectivas que pueden complicar los programas de control tradicionales en las áreas de introducción. Estos programas de vigilancia para la detección de poblaciones de insectos exóticos deben tener carácter permanente, sistemático y periódico, al tiempo que permite la detección de posibles cambios en la abundancia y distribución de dichas especies y de aquellas nativas con las cuales pudieran competir. Por tanto, también resulta necesario conocer lo más que se pueda la biología y ecología de las especies para entender con ello posibles cambios anormales de la estructura y funcionalidad de los ecosistemas.

La modelación del nicho ecológico de las especies que se introducen en un territorio permitiría mediante sistemas de información geográfica determinar áreas bajo el riesgo de infestación en las cuales se podría focalizar la atención para los programas de vigilancia y detección oportuna de especies invasivas (Hay et al., 2007). La filogeografía podría ilustrar sobre la distribución histórica y actual de una especie en particular y la variabilidad genética de sus poblaciones en lugares donde han sido introducidas respecto a la población parental.

Las especies que en este trabajo se enlistan tienen implicaciones en la salud del humano, de los animales domésticos y probablemente de los animales silvestres. Se debe establecer su posible presencia en los diferentes ecosistemas y estimar su importancia en la salud pública y animal regional y, en caso de ser invasoras, determinar el efecto de su presencia en la funcionalidad de los ecosistemas nativos, aspectos sobre los cuales se apoya la calidad de vida de la población humana y la economía de una nación. Creemos que la adición de especies a la lista de dípteros exóticos en México es un apoyo valioso a la Estrategia Nacional sobre Especies Invasoras en México a la vez que se fomenta el interés por campos de la biología que hoy día son poco valorados.

\section{Conclusiones}

En este trabajo se dan a conocer 20 especies de insectos dípteros exóticos con interés médico y veterinario en México y se presentan datos adicionales sobre las dos especies previamente consideradas en el Acuerdo por el que se determina la Lista de las Especies Exóticas Invasoras para México y la importancia sanitaria que cada una tiene. Se hace patente que para el caso de las especies con interés médico y veterinario exóticas existe una relación con el grado de sinantropía al tiempo que se definen diferentes aspectos que deben considerarse para la valoración de las especies invasivas. Se enfatiza la importancia de la taxonomía y de los estudios faunísticos elaborados con base en capturas sistemáticas de largo plazo para contar con inventarios faunísticos que desafortunadamente aún son muy escasos en México; de este modo, registrar las especies exóticas y de entre ellas distinguir las que son invasoras. Los estudios faunísticos y taxonómicos son la base para después reconocer las perturbaciones que dichas intromisiones pueden causar en la funcionalidad de los ecosistemas naturales. Las universidades, centros de investigación y revistas científicas del país no deben desestimar la importancia de estos temas que son la base no sólo para la detección de especies exóticas, sino para conducir cualquier estudio de ecología de poblaciones y de comunidades, así como para determinar su impacto en la salud humana y animal. Todas las especies de la lista tienen interés desde el punto de vista médico, sanitario, agropecuario y ecológico, por lo que su importancia económica, aun cuando no se haya evaluado, es mayúscula.

\section{REFERENCIAS}

Bhatt, S., Gething, P. W., Brady, O. J., Messina, J. P., Farlow, A. W., Moyes, C. L., Drake, J. M., Brownstein, J. S., Hoen, A. G., Sankoh, O., Myers, M. F., George, D. B., Jaenisch, T., Wint, G. R., Simmons, C. P., Scott, T. W., Farrar, J. J., \& Hay, S. I. (2013). The global distribution and burden of dengue. Nature, 496, 504-507. https://doi.org/10.1038/nature12060 
Bishop, F. (1913). The Stable Fly (Stomoxys caclitrans L.). An important livestock pest. Journal of Economic Entomology, 6, 112-126.

Borkent, A., Brown, B. V., Adler, P. H., Amorim, D. S., Barber, K., Bickel, D., Boucher, S., Brooks, S. E., Burger, J. (...) Zumbado, M. A. (2018). Remarkable fly (Diptera) diversity in a patch of Costa Rican cloud forest: Why inventory is a vital science. Zootaxa, 4402, 53-90. https://doi.org/10.11646/zootaxa.4402.1.3

Brady, O. J., Gething, P. W., Bhatt, S., Messina, J. P., Brownstein, J. S., Hoen, A. G., Moyes, C. L., Farlow, A. W., Scott, T. W., \& Hay, S.I. (2012). Refining the global spatial limits of dengue virus transmission by evidence-based consensus. PLoS Neglected Tropical Diseases, 6. https://doi.org/10.1371/journal.pntd.0001760

Brown, B. V., Marshall, S. A., \& Wood, D. M. (2009). Natural history. In B. V. Brown, A. Borkent, J. M.Cumming, N. E.Woodley, \& M. A. Zumbado. Manual of Central American Diptera (pp. 51-63). Ottawa: NRC Research Press.

Chillcott, J. G. (1961). A revision of the Nearctic species of Fanniinae (Diptera: Muscidae). The Canadian Entomologist, 14, 1-295. https://doi.org/10.4039/entm9214fv

Comité Asesor Nacional sobre Especies Invasoras. (2010). Estrategia nacional sobre especies invasoras en México, prevención, controly erradicación. México: CONABIO, Comisión Nacional de Áreas Protegidas, Secretaría de Medio Ambiente y Recursos Naturales.

CONABIO (Comisión Nacional para el Conocimiento y Uso de la Biodiversidad). (2019). Búsqueda de especies exóticas invasoras. Disponible en http://www.biodiversidad.gob.mx/especies/Invasoras/especies.html (consultado el 2 de octubre de 2019).

Dear, J. P. (1985). A revision of the new world Chrysomyini (Diptera: Calliphoridae). Revista Brasileira de Zoologia, 13, 109-169. https://doi.org/10.1590/S010181751985000300001

Diario Oficial de la Federación. (2016). Acuerdo por el que se determina la Lista de las Especies Exóticas Invasoras para México. Diario Oficial de la Federación. Disponible en http://www.dof.gob.mx/nota_detalle.php?codigo $=5464456 \&$ fecha $=07 / 12 / 2016$

Di Castri, F., Hansen, A. J., \& Debussche, M. (1990). Biological invasions in Europe and the Mediterranean Basin. Springer.

Expósito Martín, F. J. (2012). La globalización. Concepto, características, factores y consecuencias de la "Aldea global”. Temas para la Educación. Revista Digital para Profesores de la Enseñanza, 16, 1-9. Disponible en https://www.feandalucia.ccoo.es/docu/p5sd8996.pdf

Gillespie, B. E., Owens, W. E., Nickerson, S. C., \& Oliver, S. P. (1999). Deoxyribonucleic acid fingerprinting of Staphylococcus aureus from heifer mammary secretions from horn flies. Journal of Dairy Science, 82, 1581 1585. https://doi.org/10.3168/jds.S0022-0302(99)75386-5.

Greenberg, B. (1973). Flies and diseases: II. Biology and disease transmission. New Jersey: Princeton University Press.

Greenberg, B. (1988). Chrysomya megacephala (F.) (Diptera: Calliphoridae) collected in North America and notes on Chrysomya species present in the New World. Journal of Medical Entomology, 25, 199-200. https://doi.org/10.1093/jmedent/25.3.199

Grimaldi, D., \& Engel, M. S. (2005). Evolution of the insects. New York: Cambridge University Press.

Hawley, W. A. (1988). The biology of Aedes albopictus. Journal of the American Mosquito Control Association, 1, 1-39.

Hawley, W. A., Reiter, P., Copeland, R. S., Pumpuni, C. B., \& Craig Jr., G. B. (1987). Aedes albopictus in North America: Probable introduction in used tires from northern Asia. Science, 236, 1114-1116. https://doi. org/10.1126/science.3576225 
Hay, S. I., Graham, A. J., \& Rogers, D. J. (2007). Global mapping of infectious diseases: methods, examples, and emerging applications. London: Academic Press.

Hogsette, J. A., \& Farkas, R. (2000). Secretophagous and haematophagous higher Diptera. In L. Papp, \& B. Darvas (Eds.), Manual of Palaearctic Diptera. Volume 1. General and Applied Dipterology (pp. 769-792). Budapest: Science Herald.

Hövemeyer, K. (2000). Ecology of Diptera. In L. Papp, \& B. Darvas (Eds.). Manual of Palaearctic Diptera. Volume 1. General and Applied Dipterology (pp. 437-489). Budapest: Science Herald.

Ibáñez-Bernal, S. (2000). Psychodidae (Diptera), en J. Llorente-Bousquets, E. González-Soriano y N. Papavero (Comps.), Biodiversidad, Taxonomía y Biogeografía de Artrópodos de México: Hacia una sintesis de su conocimiento. Volumen II. (pp. 607-626). México. UNAM.

Ibáñez-Bernal, S. (2008). New records and descriptions of Mexican moth flies (Diptera: Psychodidae). Transactions of the American Entomological Society, 134, 87-131.

Ibáñez-Bernal, S. (2012). Zoonosis transmitidas por artrópodos: enfermedades emergentes y re-emergentes de actualidad. MEDLAB, 4, 4-16.

Ibáñez-Bernal, S. (2017). Actualización del Catálogo de Autoridades Taxonómicas de los dípteros (Diptera: Insecta) de México. Instituto de Ecología A.C. Red Ambiente y Sustentabilidad. Informe final-SNIBCONABIO proyecto JE006. Disponible en http://www.conabio.gob.mx/institucion/proyectos/ resultados/InfJE006.pdf

Ibáñez-Bernal, S., \& Martínez-Campos, C. (1994). Aedes albopictus in Mexico. Journal of the American Mosquito Control Association, 10, 231-232.

IUCN (International Union for Conservation of Nature). (2019). Global invasive data base. Species Survival Commission. Retrieved from: http://issg.org/database/species/search.asp?st=sss\&sn=\&rn=Mexico\&ri=18917\&hci=-1\&ei=-1\&fr=1\&sts=\&lang=EN

James, M. T. (1947). The flies that cause myiasis in man. Miscellaneous Publication. United States Department of Agriculture, 631, 1-175.

Larroza, M. (2013). Caracterización de la melofagosis en ovinos en la región patagónica: ciclo biológico, dinámica poblacional y distribución. Retrieved from: http://sedici.unlp.edu.ar/handle/10915/34476

Larsen, J. R., \& Ashley, R. F. (1971). Demonstration of Venezuelan equine encephalomyelitis virus in tissues of Aedes aegypti. American Journal of Tropical Medicine and Hygiene, 20, 754-760.

León Montoya, G., Sánchez, R. A., David, J., \& Wolff, E. M. (2009). Sinantropía de Calliphoridae (Diptera) del Municipio La Pintada, Antioquia-Colombia. Revista Colombiana de Entomología, 35, 73-82. Retrieved from http://www.scielo.org.co/scielo.php?script=sci_arttext\&pid=S0120-04882009000100014\&ln$\mathrm{g}=\mathrm{en} \& \mathrm{t} \operatorname{lng}=\mathrm{es}$.

Martínez-Rojano, H., Noguez, J. C., \& Huerta, H. (2018). Nosocomial myiasis caused by Lucilia sericata (Diptera: Calliphoridae) and neonatal myiasis by Sarcophaga spp. (Diptera: Sarcophagidae) in Mexico. Case Reports in Infectious Diseases. https://doi.org/10.1155/2018/5067569

Mayr, E. (1969). Principles of Systematic Zoology. New York: McGraw-Hill.

Nuorteva, P. (1963). Synanthropy of blowflies (Dipt., Calliphoridae) in Finland. Annales Entomologici Fennici, 29, 1-49.

Pape, T. (2009). Economic importance of Diptera. In B. V. Brown, A. Borkent, J. M. Cumming, D. M. Wood, N. E. Woodley, \& M. A. Zumbado, Manual of Central American Diptera (pp. 65-77). Ottawa: NRC-CNRC Research Press. 
Pape, T., Blagoderov, V., \& Mostovski, M. B. (2011). Order Diptera Linnaeus, 1758. In Z. -Q. Zhang (ed.), Animal biodiversity: An outline of higher-level classification and survey of taxonomic richness. Zootaxa, $3148,1-237$.

Pape, T., \& Evenhuis, N. (2019). Systema Dipterorum. The biosystematic data base of world Diptera. Retrieved from http://sd.zoobank.org/Nomenclator

Pimentel, D., Zúñiga, R., \& Morrison, D. (2005). Update on the environmental and economic costs associated with alien-invasive species in the United States. Ecological Economics, 52, 273-288.

Povolnỳ, D. (1971). Synanthropy. In B. Greenberg (Ed.), Flies and diseases. Vol. I. Ecology, classification, and biotic associations (pp. 17-54). New Jersey: Princeton University Press.

Rozkošný, R., Gregor, F., \& Pont, A. C. (1997). The European Fanniidae (Diptera). Institute of Landscape Ecology. Czech Republic.

Sartre, J. P. (1947). Las moscas. México: Alianza Editorial, Editorial Patria, S. A. de C. V.

Secretariado de la Comisión para la Cooperación Ambiental. (1993). Acuerdo de Cooperación Aambiental de América del Norte. Disponible en http://www.paot.org.mx/leyes/Biblioteca/15_Acuerdo_Cooperacion.pdf

Silva, J. O. de Almeida, Carvalho-Filho, F. S., Esposito, M. C., \& Reis, G. A. (2012). First record of Chrysomya rufffacies (Macquart) (Diptera, Calliphoridae) from Brazil. Revista Brasileira de Entomologia, 56, 115-118. https://doi.org/10.1590/S0085-56262012000100019

Suaya, J. A., Shepard, D. S., Siquiera, J. B., Martelli, C. T., Lum, L. C. S., Tan, L. H., Kongsin, S., Jiamton, S., Garrido, F. (...) Hals, S. B. (2009). Cost of dengue cases in eight countries in the Americas and Asia: A prospective study. American Journal of Tropical Medicine and Hygiene, 80, 846-855. https://doi.org/10.4269/ ajtmh.2009.80.846

Wagner, R., \& Ibáñez-Bernal, S. (2009). Psychodidae (sand flies, and moth or owl flies). In B. V. Brown, A. Borkent, J. M. Cumming, D. M. Wood, N. E.Woodley, M. A. Zumbado (Eds.), Manual of Central American Diptera. Vol. 1, Ottawa: NRC Research Press.

WHO (World Health Organization). (2019). Dengue and severe dengue. Retrieved from: https://www.who.int/ news-room/fact-sheets/detail/dengue-and-severe-dengue

Wiegmann, B. M., Trautwein, M. D., Winkler, I. S., Barr, N. B., Kim, J. -W., Lambkin, C., Bertone, M. A., Cassel, B. K., Bayless, K. M., Heimberg, A. M. (...) Yeates, D. K. (2011). Episodic radiations in the fly tree of life. Proceedings of the National Academy of Sciences U. S. A., 108, 5690-5695. https://doi.org/10.1073/ pnas. 1012675108

Whitworth, T. L. (2006). Keys to the genera and species of blow flies (Diptera: Calliphoridae) of America north of Mexico. Proceedings of the Entomological Society of Washington, 108, 689-725.

Whitworth, T. L. (2014). A revision of the Neotropical species of Lucilia Robineau-Desvoidy (Diptera: Calliphoridae).Zootaxa, 3810, 1-76. https://doi.org/10.11646/zootaxa.3810.1.1

Wilcove, D. S., Rothstein, D., Dubow, J., Phillips, A., \& Losos, E. (1998). Quantifying threats to imperiled species in the United States. Bioscience, 48, 607-615.

Yan, L., Pape, T., Elgar, M. A., Gao, Y., \& Zhang, D. (2019). Evolutionary history of stomach bot flies in the light of mitogenomics. Systematic Entomology. https://doi.org/10.1111/syen.12356

Zumpt, F. (1965). Myiasis in man and animal in the Old World. London: Butterwords.

\section{BY-NC-ND}

